\title{
Photoelectron Diffraction Imaging of a Molecular Breakup Using an X-Ray Free-Electron Laser
}

Gregor Kastirke, ${ }_{1}^{1}$ Markus S. Schöffler, ${ }^{1}$ Miriam Weller, ${ }^{1}$ Jonas Rist, ${ }^{1}$ Rebecca Boll, ${ }^{2}$ Nils Anders, ${ }^{1}$ Thomas M. Baumann, ${ }^{2}$ Sebastian Eckart, ${ }^{1}$ Benjamin Erk, ${ }^{3}$ Alberto De Fanis, ${ }^{2}$ Kilian Fehre, ${ }^{1}$ Averell Gatton, ${ }^{4}$ Sven Grundmann, ${ }^{1}$ Patrik Grychtol, ${ }^{2}$ Alexander Hartung, ${ }_{2}^{1}$ Max Hofmann, ${ }_{1}^{1}$ Markus Ilchen, ${ }^{2,5}$ Christian Janke, ${ }^{1}$ Max Kircher, ${ }^{1}$ Maksim Kunitski, ${ }^{1}$ Xiang Li ${ }^{6}$ Tommaso Mazza, ${ }^{2}$ Niklas Melzer, ${ }^{1}$ Jacobo Montano, ${ }^{2}$ Valerija Music, ${ }^{2,5}$ Giammarco Nalin, ${ }^{1}$ Yevheniy Ovcharenko, ${ }^{2}$ Andreas Pier, ${ }^{1}$ Nils Rennhack, ${ }^{2}$ Daniel E. Rivas, ${ }^{2}$ Reinhard Dörner, ${ }^{1}$ Daniel Rolles, ${ }^{6}$ Artem Rudenko, ${ }^{6}$ Philipp Schmidt, ${ }^{2,5}$ Juliane Siebert, ${ }^{1}$ Nico Strenger, ${ }^{1}$ Daniel Trabert, ${ }^{1}$ Isabel Vela-Perez, ${ }^{1}$ Rene Wagner, ${ }^{2}$ Thorsten Weber, ${ }^{7}$ Joshua B. Williams, ${ }^{8}$ Pawel Ziolkowski, ${ }^{2}$ Lothar Ph. H. Schmidt, ${ }_{1}^{1}$ Achim Czasch, ${ }^{1}$ Florian Trinter, ${ }^{3,9}$ Michael Meyer, ${ }^{2}$ Kiyoshi Ueda, ${ }^{10}$ Philipp V. Demekhin, ${ }^{, *}$ and Till Jahnke ${ }^{1, \dagger}$

${ }^{1}$ Institut für Kernphysik, Goethe-Universität, Max-von-Laue-Strasse 1, 60438 Frankfurt am Main, Germany

${ }^{2}$ European XFEL GmbH, Holzkoppel 4, 22869 Schenefeld, Germany ${ }^{3}$ Deutsches Elektronen-Synchrotron (DESY), Notkestrasse 85, 22607 Hamburg, Germany

${ }^{4}$ SLAC National Accelerator Laboratory, Menlo Park, California 94025, USA

${ }^{5}$ Institut für Physik und CINSaT, Universität Kassel, Heinrich-Plett-Strasse 40, 34132 Kassel, Germany

${ }^{6} J$. R. Macdonald Laboratory, Department of Physics, Kansas State University, Manhattan, Kansas 66506, USA

${ }^{7}$ Lawrence Berkeley National Laboratory, Chemical Sciences and Ultrafast X-ray Science Laboratory, Berkeley, California 94720, USA

${ }^{8}$ Department of Physics, University of Nevada, Reno, Nevada 89557, USA

${ }^{9}$ Molecular Physics, Fritz-Haber-Institut der Max-Planck-Gesellschaft, Faradayweg 4-6, 14195 Berlin, Germany

${ }^{10}$ Institute of Multidisciplinary Research for Advanced Materials, Tohoku University, Sendai 980-8577, Japan

(Received 5 November 2019; revised manuscript received 30 March 2020; accepted 15 April 2020; published 8 June 2020)

A central motivation for the development of $\mathrm{x}$-ray free-electron lasers has been the prospect of timeresolved single-molecule imaging with atomic resolution. Here, we show that $\mathrm{x}$-ray photoelectron diffraction-where a photoelectron emitted after x-ray absorption illuminates the molecular structure from within — can be used to image the increase of the internuclear distance during the $\mathrm{x}$-ray-induced fragmentation of an $\mathrm{O}_{2}$ molecule. By measuring the molecular-frame photoelectron emission patterns for a two-photon sequential $K$-shell ionization in coincidence with the fragment ions, and by sorting the data as a function of the measured kinetic energy release, we can resolve the elongation of the molecular bond by approximately 1.2 a.u. within the duration of the x-ray pulse. The experiment paves the road toward timeresolved pump-probe photoelectron diffraction imaging at high-repetition-rate $\mathrm{x}$-ray free-electron lasers.

DOI: $10.1103 /$ PhysRevX.10.021052

Subject Areas: Atomic and Molecular Physics

\section{INTRODUCTION}

Following chemical reactions and structural changes in molecules in real time (and eventually steering and controlling these dynamics) has been a long-standing dream in physical chemistry. Accordingly, time-resolved

\footnotetext{
*demekhin@physik.uni-kassel.de

†jahnke@atom.uni-frankfurt.de
}

Published by the American Physical Society under the terms of the Creative Commons Attribution 4.0 International license. Further distribution of this work must maintain attribution to the author(s) and the published article's title, journal citation, and DOI. single-molecule imaging has been one central aim triggering the development of x-ray free-electron lasers (XFELs). The intent of this new class of time-resolved imaging approaches is to investigate geometrical changes of a molecule with atomic resolution in real time, which is complementary to standard femtochemistry [1] approaches that follow the potential energy landscape of a molecule. Photoelectron diffraction is one of the envisioned techniques among others [2-5]. The idea of x-ray-induced photoelectron diffraction imaging as an analytical tool is already well established in surface sciences [6]. Almost two decades ago it was pointed out that this technique can be used in the gas phase for deeply bound (i.e., localized) innershell electrons [7]. With the advent of XFELs, corresponding studies in the gas phase 
came into reach [8-11]. Detailed information can be retrieved when the electron angular-emission distribution in the molecular frame of reference is obtained, which is the key to photoelectron diffraction imaging in the gas phase. Upon photoabsorption, an electron wave is launched from a well-defined location in the molecule, thus "illuminating the molecule from within" [7]. Its angular distribution is a manifestation of a complex interference pattern of this electron wave as it is multiple scattered by the molecular potential on its way out. Such distributions show very rich features depending on the molecular species under investigation, the initial orbital the electron is emitted from, the polarization, as well as the wavelength of the ionizing photons. In particular, polarization-averaged molecularframe electron angular distributions have been recently demonstrated to capture many molecular details [12], such as the internuclear distance at the instant of emission [13] or even the whole molecular structure as such [14]. In order to observe molecular-frame electron angular distributions, the orientation of the molecule at the instant of photoionization needs to be known. This is achieved by either actively aligning molecules in the gas phase via laser pulses [15] or by determining their orientation a posteriori from a coincident measurement of the ionic fragments, which are in many cases produced after innershell photoionization of a molecule $[7,14,16]$. The high repetition rate of the European XFEL finally opens the door to such coincidence experiments and we present a first successful implementation of this technique using a COLTRIMS reaction microscope $[17,18]$ at the European XFEL. Because of the high photon density in the focus of the XFEL beam, several photons can be absorbed sequentially by an oxygen molecule within a single light pulse. Absorption of the first photon and subsequent Auger decay triggers the breakup of the dication in a Coulomb explosion. During elongation of the molecular bond in the dissociation process, the molecule can absorb a second photon and emit another $K$-shell electron, which is then used for the photoelectron diffraction imaging in our experiment. The photons have energies of approximately $670 \mathrm{eV}$. We are able to obtain internuclear distance-resolved photoelectron diffraction patterns, investigating the aforementioned naturally occurring fragmentation of the molecule upon $K$-shell ionization during the duration of the x-ray pulse of approximately 25 fs. Our experiment demonstrates that photoelectron diffraction imaging is finally possible using high repetition rate XFELs. In particular, it paves the road toward time-resolved pump-probe photoelectron diffraction imaging.

\section{EXPERIMENT}

In our experiment we employ soft $\mathrm{x}$-ray pulses at the Small Quantum Systems (SQS) instrument at the European XFEL [19] to generate multiple core holes in $\mathrm{O}_{2}$ molecules. A COLTRIMS reaction microscope [18] installed as a permanent end station has been used for the coincident measurement of all generated molecular fragments. Details on the experimental setup and the x-ray properties can be found in the Appendix A.

A scheme of the reaction triggered is shown in Fig. 1: A first photon is absorbed ionizing an $\mathrm{O}_{2} K$-shell electron. Subsequently, an Auger decay causes the breakup of the molecule in a Coulomb explosion. During the dissociation of the molecule a second photon may ionize a further $K$ shell electron. This electron is used to illuminate the fragmenting molecule from within. By measuring the finally occurring singly and triply charged atomic ions and the second photoelectron in coincidence, we are able to perform internuclear distance-resolved photoelectron diffraction imaging.

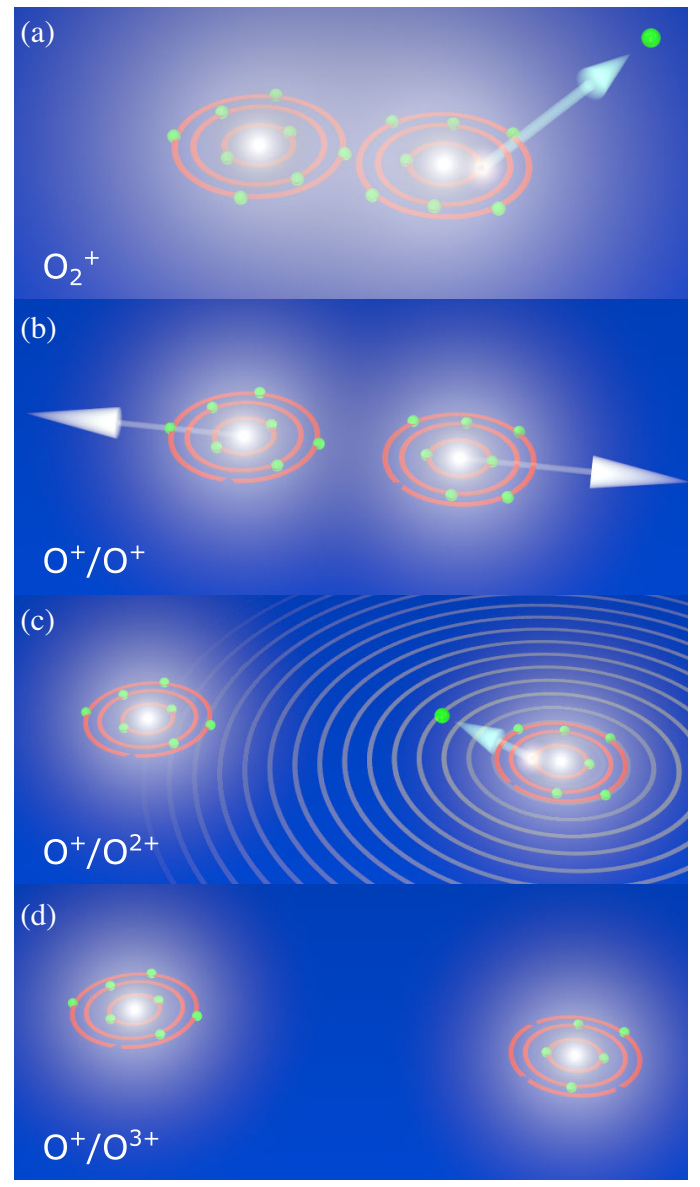

FIG. 1. Scheme of photoelectron diffraction imaging during Coulomb explosion of $\mathrm{O}_{2}$. (a) A $K$-shell electron is ionized upon irradiation with XFEL light. (b) After the innershell vacancy has been filled after a first Auger decay, the molecule is doubly charged and fragments in a Coulomb explosion. (c) During the fragmentation a second photon triggers the emission of another $K$-shell electron illuminating the molecule from within. (d) A further, subsequent Auger decay yields the observed final $\mathrm{O}^{+} / \mathrm{O}^{3+}$ state in which the Coulomb explosion continues until the ions are well separated and finally detected. Steps (a) to (c) occur during a single XFEL light pulse, i.e., within approximately 25 fs. 
As the absorption of the second photon occurs at random delay times during the light pulse, the information on the delay between the two photoabsorption events needs to be extracted from other observables in our coincidence measurement. In the example of $\mathrm{H}_{2}$, it is possible to obtain information on the internuclear distances from the kinetic energy of the ionic fragments after a Coulomb explosion, as this energy depends only on the initial internuclear distance of the two protons [20]. However, in the present study on oxygen, the relation between interatomic distance and kinetic energy release (KER) is not straightforward: Two sequential innershell photoabsorptions with subsequent Auger decay yield a total charge state of four, thus involving four excited-state potential energy curves. A simplified sketch of the involved charge states is shown in Fig. 2. This sketch neglects the comparably complex electronic structure of $\mathrm{O}_{2}$, and for example, in the case of a breakup into $\mathrm{O}^{+} / \mathrm{O}^{+}$after core ionization the KER spectrum exhibits already several distinct features that have been attributed to a multitude of different decay

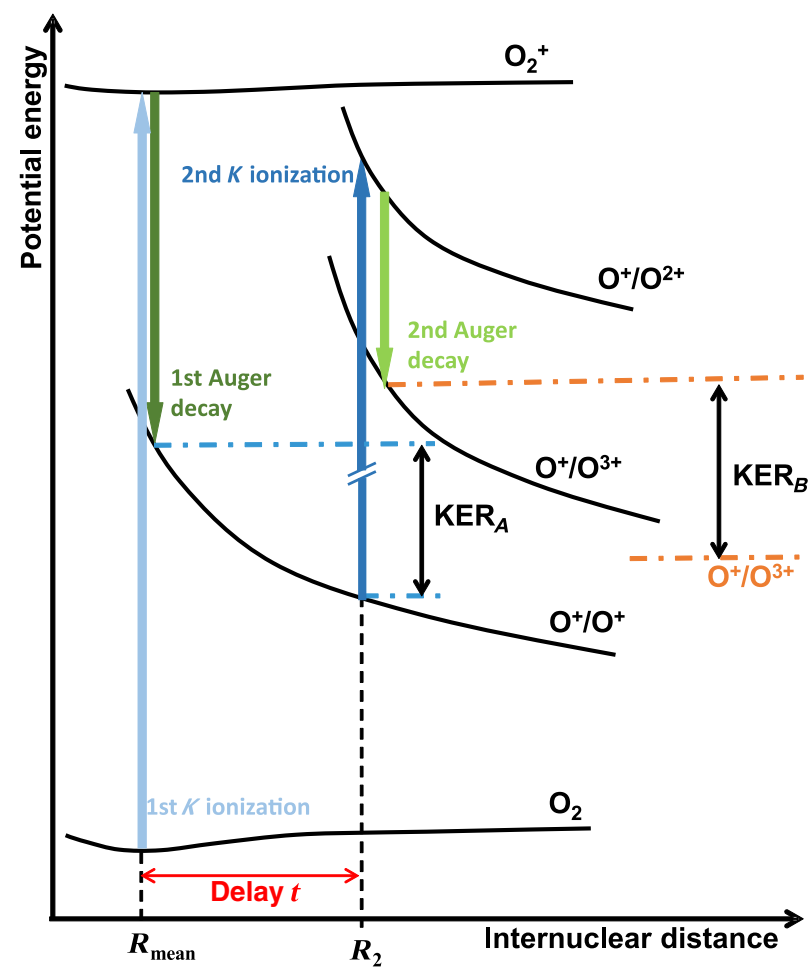

FIG. 2. Schematic of the modeled internuclear distance extraction. After initial $K$-shell ionization of the neutral molecule at the mean internuclear distance $R_{\text {mean }}$, an Auger decay of the singly charged molecular ion triggers a Coulomb explosion along the repulsive $\mathrm{O}^{+} / \mathrm{O}^{+}$curve. The second $K$-shell electron is ionized at internuclear distance $R_{2}$ after a delay $t$. The molecule is now in an $\mathrm{O}^{+} / \mathrm{O}^{2+}$ state, which—within our simplified modelit immediately leaves after emission of a second Auger electron. The Coulomb explosion continues along the $\mathrm{O}^{+} / \mathrm{O}^{3+}$ repulsive curve. The total kinetic energy of the ions $\mathrm{KER}=\mathrm{KER}_{A}+$ $\mathrm{KER}_{B}$ is measured in our experiment. pathways toward several final $\mathrm{O}^{+} / \mathrm{O}^{+}$states $[21,22]$. Thus, a direct extraction of the internuclear distance from the measured KER of individual quadruple ionization events is expected to be futile. We therefore pursue a different path for our analysis and focus first on polarization-averaged molecular-frame photoelectron angular distributions obtained from a full theoretical modeling in order to compare these to the ones obtained from our experiment.

\section{THEORY}

For linearly polarized ionizing radiation, the angularemission distribution of photoelectrons in the frame of a diatomic molecule is given (within the dipole approximation) by

$$
\frac{d \sigma}{d \Omega}(\beta, \theta, \phi)=\left|\sum_{l m k}(-i)^{l} D_{k 0}^{1}(\beta) A_{\text {clmk }} Y_{l m}(\theta, \phi)\right|^{2} .
$$

Here, $\beta$ is the angle between the molecular axis and the direction of the polarization of the ionizing radiation (the remaining orientation Euler angles $\alpha$ and $\gamma$ are set to zero), $\theta$ and $\phi$ are the photoelectron emission angles defined with respect to the molecular axis, $Y_{l m}$ are spherical harmonics, and $D_{k 0}^{1}$ are the Wigner rotation matrices. Electron dynamics of the considered process is imprinted in the amplitudes $A_{\text {elmk }}$ for the emission of the partial electron continuum waves of energy $\epsilon$ with the angular momentum quantum numbers $l$ and $m$ through the absorption of one photon of polarization $k=0 \pm 1$, as defined in the frame of the molecule.

Within our theoretical model, the photoionization transition amplitudes $A_{\text {elmk }}$ are computed by using the stationary single-center method and code [23-25], which provide an accurate theoretical description of the angleresolved photoemission spectra of molecules. The calculations are performed in the relaxed-core Hartree-Fock approximation enabling relaxation of the molecular orbitals in the field of the core vacancy. The single-center expansion of all occupied orbitals of $\mathrm{O}_{2}$ with respect to the geometrical center of the molecule is restricted to partial harmonics with $l \leq 99$, and for the photoelectron in the continuum by $l \leq 49$ (note that in diatomic molecules, the projection $m$ of the angular momentum $l$ is a well-defined quantum number).

For the first photoelectron, the neutral $\mathrm{O}_{2}$ molecule in the initial photoionization step is considered to be in its ground electronic state at the respective equilibrium internuclear distance. Calculations are performed for a photoelectron kinetic energy of $\epsilon=127 \mathrm{eV}$ and averaged over the two contributions from the degenerate initial orbitals $1 \sigma_{g / u}$ (or $\left.1 s_{R / L}\right)$. For the second photoelectron, the doubly ionized $\mathrm{O}_{2}^{2+}$ molecule after the initial photoionization step is considered to be in the ground electronic state of the dication. These calculations are performed for a kinetic 

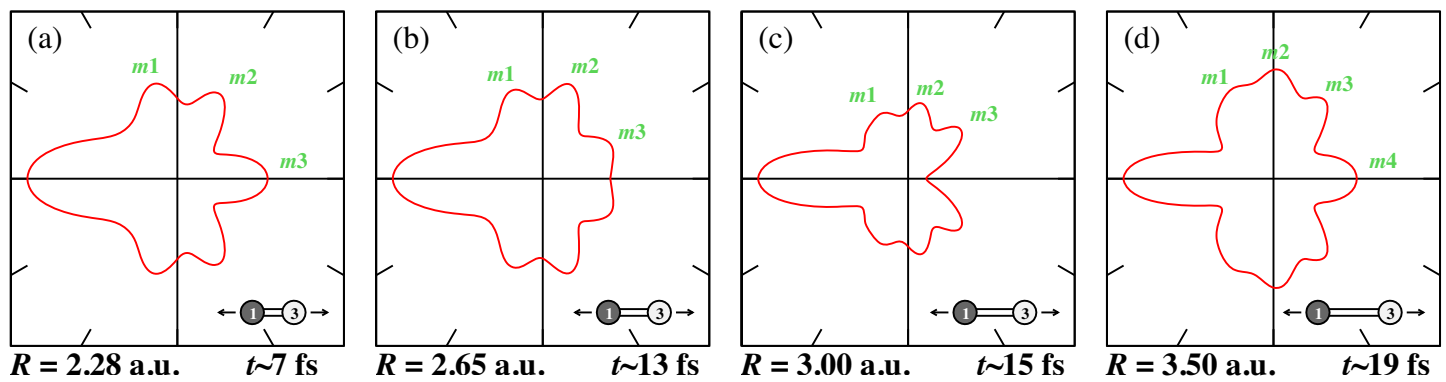

FIG. 3. Polarization-averaged molecular-frame angular distributions of the second photoelectron emitted after the first Auger decay for different internuclear distances as obtained from our theoretical calculations. Distributions ranging from (a) $R=2.28$ a.u. (mean ground state internuclear distance) to (d) $R=3.50$ a.u., which is the internuclear distance that can be reached within the duration of the XFEL pulse, are shown. The second photon has been absorbed by the ion on the right. An electron energy of $93 \mathrm{eV}$ is used for the simulations. The labels $m 1$ to $m 4$ depict the different maxima mentioned in the text. The distributions are symmetric by definition with respect to the molecular axis.

energy of $\epsilon=93 \mathrm{eV}$ and several internuclear distances, as indicated in Fig. 3. Only the contributions from the initial orbital $1 s_{R}$, which is localized at the oxygen atom being triply charged at the end, is considered. In both cases, angular-emission distributions are computed in the orientation interval $\beta \in\left[0^{\circ}, 180^{\circ}\right]$ in steps of $\Delta \beta=1^{\circ}$ and then averaged over these orientations of the polarization axis of the light with respect to the molecular axis.

\section{RESULTS AND DISCUSSION}

We can distinguish the first photoelectron stemming from the pump and the second originating from the probe step from the measured electron energy. At the photon energy employed in our experiment $(h \nu=670 \mathrm{eV})$, the first photoelectron is emitted at an electron energy of $127 \mathrm{eV}$, the second within a range of $80 \mathrm{eV}<E_{e}<$ $105 \mathrm{eV}$ depending on the emission time. As a check of the validity of our modeling, we examine the results obtained for the first $K$-shell electron (Fig. 4). The angular distribution of this photoelectron-emitted in the pump step-is fully symmetric with respect to the $\mathrm{O}^{+}$and $\mathrm{O}^{3+}$ sides of the fragmentation. This highlights that the symmetry of the molecule is not yet broken during this first ionization step. The modeled molecular-frame electron angular distribution corresponds nicely to the measure one.

The theoretical predictions of the angular distributions of the photoelectrons from the probe step (i.e., the second photoionization) are depicted in Fig. 3. They are highly asymmetric. This is a direct consequence of the fact that the second photoionization is assumed to occur at the $K$ orbital localized at the right-hand oxygen ion (labeled " 3 " in the inset). The main lobe is directed toward the singly charged oxygen ion, showing the ability of photoelectron diffraction to image the neighboring atom. Inspecting the calculated distributions in Fig. 3 reveals a main trend, which is the occurrence of additional lobes and minima as the internuclear distance increases. For example, the maximum along the molecular axis on the right of Fig. 3(a) (labeled as $m 3$ ) rotates toward larger angles in Figs. 3(b) and 3(c) as a minimum occurs along the molecular axis, which finally turns into a maximum $m 4$ in Fig. 3(d). Such behavior is well known from interference-based effects (e.g., Bragg diffraction, double slit scattering), where an increase of the distance between the interfering virtual sources of the waves leads to more narrowly spaced interference fringes. For the homonuclear molecule $\mathrm{O}_{2}$, this behavior is expected as well and occurs as a direct consequence of the electron scattering process: Even though the electron emission in question can be considered to originate from the eventually triply charged ion, the electron is scattered by the other ion and the interference of direct and scattered pathways forms the observed bodyfixed electron emission pattern. For illustration, we show in Fig. 5 a sequence of corresponding angular distributions as obtained from interfering a direct and a (phase-shifted) scattered wave with wavelength $\lambda$. In this simple two-center model, the body-fixed emission pattern is characterized by

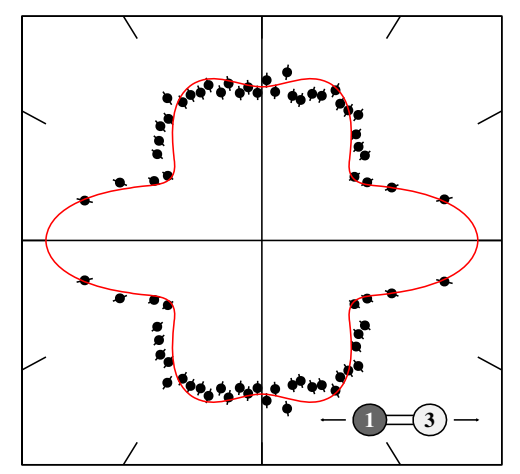

FIG. 4. Polarization-averaged molecular-frame angular distribution of the first photoelectron emitted in the overall process. The molecule is aligned horizontally with the triply charged $\mathrm{O}^{3+}$ ion located on the right. The XFEL photon energy is set to $h \nu=670 \mathrm{eV}$, yielding a $K$-shell electron of approximately $127 \mathrm{eV}$ kinetic energy. The red line is the result of our full theoretical calculations. 

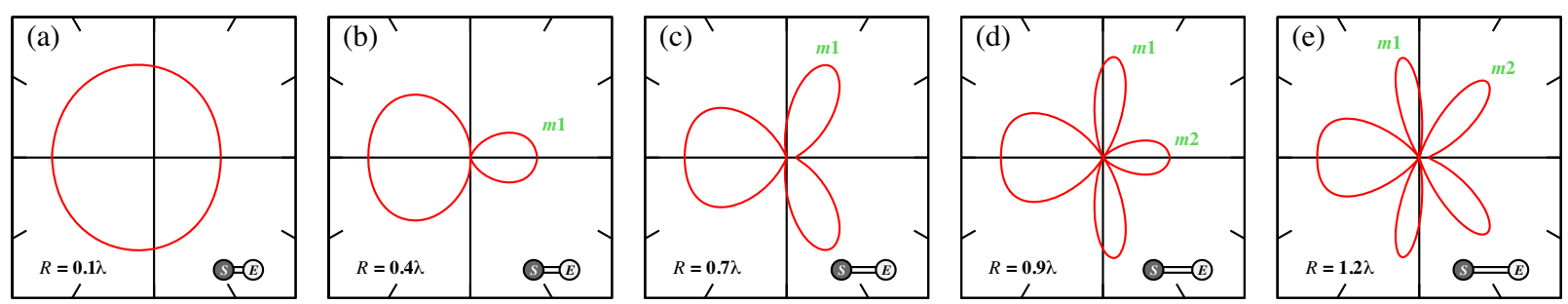

FIG. 5. Examples of diffraction patterns in polar representation. A wave with wavelength $\lambda$ is emitted from the right center (emitter, labeled " $E$ ") and diffracted at the left center (scatterer, labeled " $S$ "). The red line depicts the angular distribution of the superposition of the direct and the scattered wave. (a) to (e) show the angular distributions for different distances between $E$ and $S$. (a) At shortest distances no diffraction lobes are visible. (b) As the distance increases, a first maximum $m 1$ forms toward the emitter side. (c) Further increase yields the first minimum in the direction of $E$ and the maximum $m 1$ moves toward the main lobe in the direction of $S$. As the distance is increased, a new lobe $m 2$ appears (d) and moves toward $S$, as well (e).

the phase difference $\phi$ between the two pathways that depends on the emission angle $\theta$ :

$$
\frac{d \sigma}{d \theta} \propto \cos ^{2}[\phi(\theta) / 2]
$$

Unlike interference of two direct pathways, where

$$
\phi_{d d}(\theta)=2 \pi \frac{\cos (\theta) R}{\lambda},
$$

the phase difference between direct and scattered waves is modified by the path length difference $R$ between the two centers:

$$
\phi_{\mathrm{sd}}(\theta)=2 \pi \frac{\cos (\theta) R+R}{\lambda} .
$$

Accordingly, as a dominant feature, a forward scattering peak occurs in the direction of the scatterer $S$ in all panels of Fig. 5. Every time the distance between the emitter $E$ and the scatterer $S$ is increased by $\lambda / 2$ a new maximum emerges on the right and one further increase by $\lambda / 4$ yields a new minimum. The range of internuclear distances covered by Fig. 3 corresponds to the distances that the two point charges $(m=16, q=1)$ can reach within the duration of the XFEL pulse of approximately $25 \mathrm{fs}$, after being initialized at rest at the $\mathrm{O}_{2}$ mean internuclear distance and then driven solely by their Coulomb repulsion.

Now, we investigate the experimental, polarizationaveraged molecular-frame electron angular distributions for different KER values. We find within a large range of KERs $(28 \mathrm{eV}<\mathrm{KER}<36 \mathrm{eV})$ a good qualitative agreement with the theoretical predictions. A movie of a sweep through this region can be found in the online Supplemental Material [26], and several examples of the molecular-frame electron angular distributions from within this range are depicted in Fig. 6. The results of our theoretical modeling are overlayed as a red line. It is not surprising that the agreement is only qualitative: The calculations for this second photoelectron have been performed only for fixed (i.e., single) internuclear distances and for a fixed electron energy of $93 \mathrm{eV}$ (which is the mean energy of this electron). Moreover, only the ionization of the dicationic $O_{2}^{2+}$ ground state by the second photon has been taken into account. In the experiment, as argued before, a fixed KER does most likely not correspond to a distinct internuclear distance and, in addition, the experimental data have been integrated over the electron energy range expected for the second $K$-shell electron, which has been emitted after the first Auger decay (i.e., $80 \mathrm{eV}<\mathrm{E}_{\mathrm{e}}<105 \mathrm{eV}$ ). Moreover, the experimental data include contributions from all final Auger states being

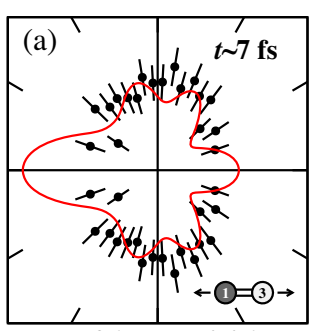

KER = 36 eV $R \sim 2.26$ a.u.

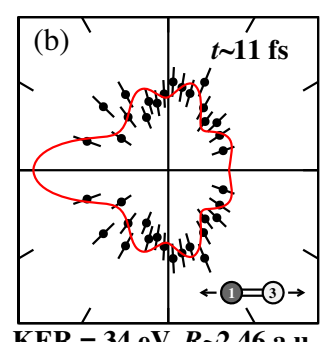

KER = $34 \mathrm{eV}$ R 2.46 a.u.

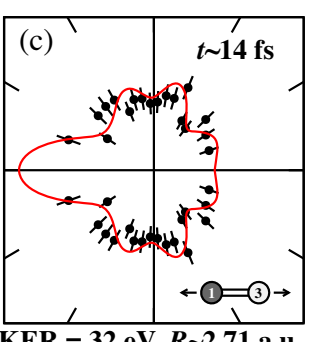

KER = 32 eV $R \sim 2.71$ a.u.

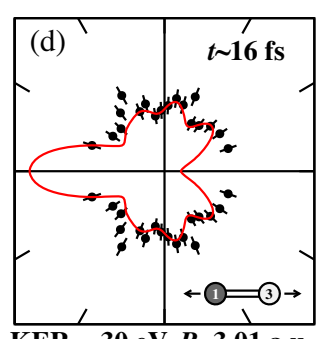

KER = 30 eV $R \sim 3.01$ a.u.

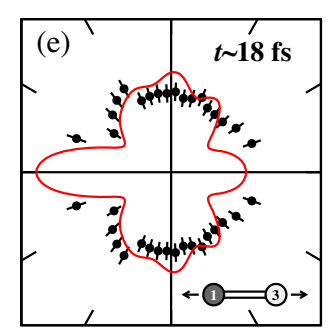

KER = 28 eV $R \sim 3.39$ a.u.

FIG. 6. Polarization-averaged molecular-frame angular distributions of the second photoelectron (emitted during the $\mathrm{O}^{+} / \mathrm{O}^{+}$ Coulomb explosion) for several KERs. The theoretical predictions shown in Fig. 3 are overlayed as red lines (see text for details). The internuclear distances $R$ and the absorption times $t$ of the second photon have been obtained using a simple Coulomb explosion model (see Appendix B). The ion, which is triply charged in the final state, is located on the right. 
ionized by the second photon, and, furthermore, the probability that the third charge created in the process remains at the oxygen ion where the electron is ejected from is not $100 \%$. Despite these conceptual flaws, the experimental distributions resemble those obtained from our theory and indicate that a similar range of internuclear distances is covered here as in the calculations (Fig. 3). This finding prompts us to employ a simplified classical model of the transitions and potential energy curves involved in the overall ionization pathway, which is described in Appendix B. This model provides values for the assumed internuclear distances computed from the corresponding KER values. The values are stated below each panel in Fig. 6, and the theoretical distribution overlaid for comparison is selected accordingly. Thus, Figs. 6(a), 6(d), and 6(e) are compared to Figs. 3(a), 3(c), and $3(\mathrm{~d})$, respectively. The internuclear distance of Fig. 3(b) is somewhat in between that belonging to the KER values employed in Figs. 6(b) and 6(c) and, accordingly, the distribution shown in Fig. 3(b) is employed for comparison to both measured angular distributions. Taking the simplicity of the model and the complexity of the $\mathrm{O}_{2}$ electronic structure into account, the level of agreement is surprisingly good, even though the measured angular distributions obtained for largest internuclear distances are more washed out. Furthermore, the measured angular distribution shown in Fig. 6(d) suggests contributions from slightly larger internuclear distances asinstead of the predicted minimum - a further lobe seems to occur in the direction of the emitting, finally triply charged $\mathrm{O}^{3+}$ ion.

\section{CONCLUSION}

Our work demonstrates that molecular-frame photoelectron diffraction imaging is a viable route for analyzing molecular dynamics while employing high-repetitionrate XFELs. Even without direct access to real-time information, the evolution of the dissociation process could be followed via scanning the KER. Remarkably, this is possible despite the multistep ionization process and revealed a first molecular movie of a sequential core-hole ionization. Upcoming two-pulse pump-probe schemes are expected to open a new realm of timeresolved studies of chemistry on the most fundamental level. With such two-pulse schemes the need of extracting geometrical information on the molecule and thus the timing of the process from ion properties, as in our case, vanishes, allowing for performing direct time-resolved photoelectron diffraction imaging. Additionally, with the future availability of femtosecond lasers synchronized to the x-ray pulses molecular dynamics can be triggered, and photoelectron diffraction imaging can be used as a powerful probe step in time-resolved experiments on molecular dynamics.

\section{ACKNOWLEDGMENTS}

We acknowledge European XFEL in Schenefeld, Germany, for provision of X-ray free-electron laser beam time at the SQS instrument and would like to thank the staff for their assistance. We are indebted to A. Bräuning-Demian for timely support during the beam-time preparations. This work has been supported by the Bundesministerium für Bildung und Forschung (BMBF), Grants No. 05K13RF4 and No. 05K16RF1. The theoretical work was supported by the DFG Project No. DE 2366/1-2. M. S. S., S. G., K. F., and R.D. acknowledge support from Deutsche Forschungsgemeinschaft via Sonderforschungsbereich 1319 $(\mathrm{ELCH})$, S. E. acknowledges support by the German Research Foundation (DFG) through priority program SPP 1840 QUTIF, M.I., P. S., and V. M. acknowledge funding by the Volkswagen foundation within a Peter Paul Ewald-Fellowship. K. U. acknowledges funding by the "Dynamic Alliance for Open Innovation Bridging Human, Environment and Materials" and the "IMRAM (Institute of Multidisciplinary Research for Advanced Materials) project." T.W. was supported by the U.S. Department of Energy under Contract No. DE-AC0205CH11231 and by the U.S. Department of Energy Office of Basic Energy Sciences, Division of Chemical Sciences, Biosciences and Geosciences. X. L, D. R., and A. R. are supported by the Chemical Sciences, Geosciences, and Biosciences Division, Office of Basic Energy Sciences, Office of Science, U.S. Department of Energy, Grant No. DE-SC0019451. J. B. W. acknowledges funding by National Science Foundation Grant No. NSF-PHY1807017.

\section{APPENDIX A: EXPERIMENTAL DETAILS AND XFEL PROPERTIES}

The experiment has been performed at the Small Quantum Systems (SQS) instrument of the European $\mathrm{X}$-Ray Free-Electron Laser in Schenefeld (Germany). A cold-target recoil-ion momentum spectroscopy (COLTRIMS) reaction microscope $[17,18]$ has been used to perform ion-ion-electron coincidence measurements of the charged fragments of $\mathrm{O}_{2}$ molecules after irradiation with femtosecond $\mathrm{x}$-ray pulses. In brief, a supersonic jet of $\mathrm{O}_{2}$ molecules has been intersected at a right angle with the ionizing light. Charged fragments have been guided by weak electric $(43.5 \mathrm{~V} / \mathrm{cm})$ and magnetic $(13.7 \mathrm{G})$ fields toward two time- and position-sensitive microchannel plate detectors with an active area of $120 \mathrm{~mm}$. The ion arm has a length of $25.5 \mathrm{~cm}$; the electron arm consists of a single acceleration region of $60.8-\mathrm{cm}$ length. From the flight times and the positions of impact the initial vector momentum of each particle can be deduced. Accordingly, the emission directions of the ionic fragments of the molecule are measured in coincidence with the photoelectron emission direction. This provides all information needed to obtain 
molecular-frame angular distributions, assuming that the axial recoil approximation holds (i.e., the diatomic molecule does not significantly rotate prior to fragmentation). The data presented in this manuscript have been obtained during a total acquisition time of approximately $17 \mathrm{~h}$.

We employ x-ray pulses with initial pulse energies of $2.4 \mathrm{~mJ}$, which are attenuated to $30 \pm 5 \mu \mathrm{J}$ by using a $15-\mathrm{m}-$ long gas absorber filled with nitrogen gas. This results in $14 \pm 2 \mu \mathrm{J}$ on target based on the calculated beam-line transmission of 0.46 at a photon energy of $670 \mathrm{eV}$. The focus size has been determined to be $\sim 0.9 \times 1.6 \mu \mathrm{m}^{2}$ at a photon energy of $1 \mathrm{keV}$ using a wave-front sensor. The EuXFEL operated at a base repetition rate of $10 \mathrm{~Hz}$, providing bursts of $\mathrm{x}$-ray pulses with a spacing of $1.1 \mathrm{MHz}$, out of which we use every $6^{\text {th }}$ pulse for our experiment, i.e., the $\mathrm{x}$-ray pulses had a spacing of $5317 \mathrm{~ns}$. We received 42 bunches per burst, i.e., $420 \mathrm{x}$-ray pulses per second. The pulse duration is not measured but is calculated to be $\sim 25$ fs based on the electron bunch charge of $250 \mathrm{pC}$.

\section{APPENDIX B: SIMPLIFIED DECAY AND COULOMB EXPLOSION MODEL}

As outlined in the main article, the complexity of the electronic structure and the overall decay path make a direct determination of the internuclear distance from the measured kinetic energy release of the ions difficult. We employ a simplified model of the ionization and decay process in order to estimate the internuclear distance at the instant of the emission of the second $K$-shell photoelectron for the movie (see online Supplemental Material [26]) and Fig. 6. Despite the simplicity of the model, the values obtained fit surprisingly well to the observed number of interference fringes and seem to confirm the assumed range of internuclear distances covered in the experiment.

A scheme of the involved potential energy curves is shown in Fig. 2. In our experiment, we concentrate on a breakup of the molecule into $\mathrm{O}^{+} / \mathrm{O}^{3+}$, which is reached by the following overall decay pathway: A Coulomb explosion is triggered, as an Auger decay occurs after the removal of the first $K$-shell photoelectron from the molecule. The system propagates along the repulsive $\mathrm{O}^{+} / \mathrm{O}^{+}$potential energy curve, which is approximated to be $1 / R$ (in atomic units) in our modeling. After an unknown delay (within the x-ray pulse duration of $25 \mathrm{fs}$ ), a second photoabsorption can take place, and the second $K$-shell electron is ionized at the internuclear distance $R_{2}$ as the molecule continues its fragmentation along the $\mathrm{O}^{+} / \mathrm{O}^{3+}$ potential energy curve (approximated by $3 / R$ ) after the emission of a second Auger electron (assumed as instantaneous). The overall kinetic energy gained within this dissociation path consists of the two contributions $\mathrm{KER}_{A}$ and $\mathrm{KER}_{B}$ as shown in Fig. 2. Given, in addition, the $\mathrm{O}^{+} / \mathrm{O}^{3+}$ potential energy curve, $R_{2}$ can be extracted from the measured total KER = $\mathrm{KER}_{A}+\mathrm{KER}_{B}$.
In order to validate that the range of internuclear distances observed in our experiment is within reach during the duration of the XFEL pulse, we simulate a Coulomb explosion of two point charges with $q=1$ that have been placed at the molecule's mean internuclear distance of $R=2.28$ a.u. without initial kinetic energy. These point charges reach internuclear distances of up to $R \sim 6.5$ a.u. within the XFEL pulse duration of approximately $25 \mathrm{fs}$. This internuclear distance, thus, should be considered as a maximum value for $R_{2}$, assuming an instantaneous Auger decay of the $\mathrm{O}_{2}^{+}$ionic state. However, considering the mean decay time of this state, the range of reachable internuclear distances reduces to approximately $R \sim 3.8$ a.u., which is still well within the range observed in our experiment. The time information provided in the movie (online Supplemental Material [26]) and Figs. 3 and 6 has been retrieved using this dissociation model along the $\mathrm{O}^{+} / \mathrm{O}^{+}$ potential energy curve, adding the mean lifetime of the decaying $\mathrm{O}_{2}^{+}$state.

A possible reason why our modeling of the chargeup and decay of the molecule yields reasonably good agreement between theory and experiment in Fig. 6 can be found in the shape of the kinetic energy release distribution. Figure 7 depicts the measured KER for (a) the $\mathrm{O}^{+} / \mathrm{O}^{+}$channel and

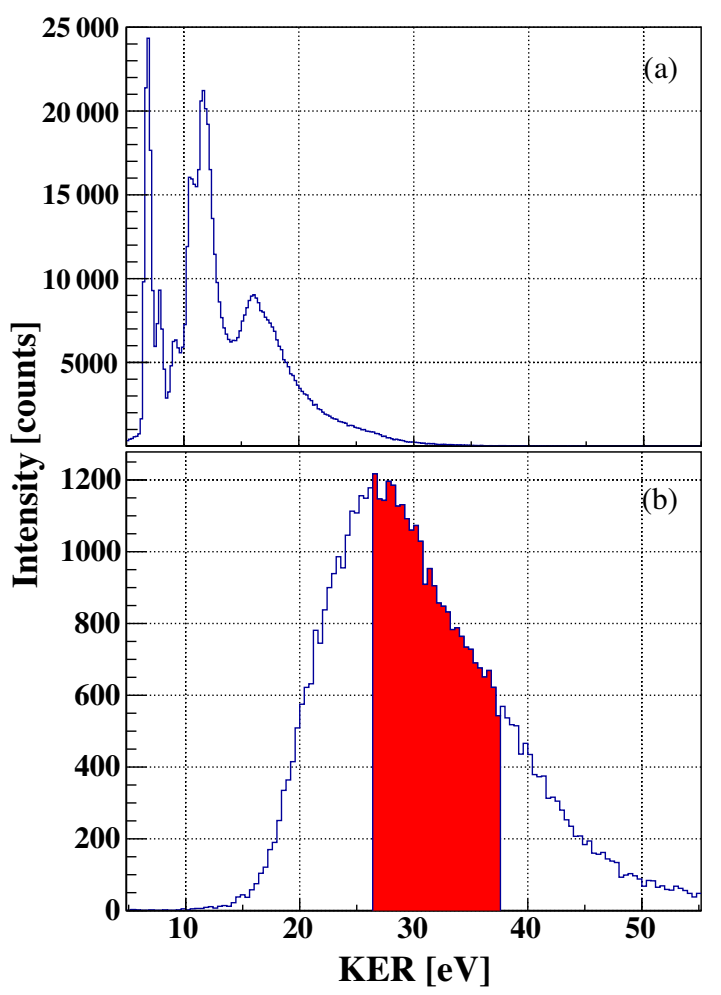

FIG. 7. Measured kinetic energy release distribution of molecules ending in (a) the doubly charged $\mathrm{O}^{+} / \mathrm{O}^{+}$final state and (b) the quadruply charged $\mathrm{O}^{+} / \mathrm{O}^{3+}$ final state. The red-marked area in (b) depicts the region of KER which is used to generate the angular distributions shown in Fig. 6. 
(b) the breakup of the molecule into $\mathrm{O}^{+} / \mathrm{O}^{3+}$. The distribution shown in Fig. 7(a) exhibits several distinct peaks resulting from intermediate $O_{2}^{+}$states, which have potential energy curves that are to some extent not repulsive. A full assignment and disentangling of these can be found in Ref. [21]. The distribution of the quadruply charged final state shown in (b) is different. It is in principle featureless and consists mainly of one single broad peak. In line with the short length of the $\mathrm{x}$-ray pulse, this suggests a scenario where high charge states are generated very rapidly, and the final kinetic energy of the ions is indeed dominated to a large extent by the Coulomb explosion along steeply repulsive potential energy curves. The KER region used to generate Fig. 6 is marked in red. The molecular-frame angular distributions corresponding to KERs outside the red region resemble those shown in Fig. 6. They show, however, no systematic evolution with respect to the KER.

[1] A. H. Zewail, Femtochemistry. Past, Present, and Future, Pure Appl. Chem. 72, 2219 (2009).

[2] T. J. A. Wolf, D. M. Sanchez, J. Yang, R. M. Parrish, J. P. F. Nunes, M. Centurion, R. Coffee, J. P. Cryan, M. Gühr, K. Hegazy et al., The Photochemical Ring-Opening of 1,3Cyclohexadiene Imaged by Ultrafast Electron Diffraction, Nat. Chem. 11, 504 (2019).

[3] R. Neutze, R. Wouts, D. van der Spoel, E. Weckert, and J. Hajdu, Potential for Biomolecular Imaging with Femtosecond X-Ray Pulses, Nature (London) 406, 752 (2000).

[4] H. Stapelfeldt, E. Constant, H. Sakai, and P. B. Corkum, Time-Resolved Coulomb Explosion Imaging: A Method to Measure Structure and Dynamics of Molecular Nuclear Wave Packets, Phys. Rev. A 58, 426 (1998).

[5] B. Wolter, M. G. Pullen, A.-T. Le, M. Baudisch, K. Doblhoff-Dier, A. Senftleben, M. Hemmer, C. D. Schröter, J. Ullrich, T. Pfeifer et al., Ultrafast Electron Diffraction Imaging of Bond Breaking in Di-Ionized Acetylene, Science 354, 308 (2016).

[6] D. P. Woodruff, Photoelectron Diffraction: From Phenomenological Demonstration to Practical Tool, Appl. Phys. A 92, 439 (2008).

[7] A. Landers, Th. Weber, I. Ali, A. Cassimi, M. Hattass, O. Jagutzki, A. Nauert, T. Osipov, A. Staudte, M. H. Prior et al., Photoelectron Diffraction Mapping: Molecules Illuminated from Within, Phys. Rev. Lett. 87, 013002 (2001).

[8] R. Boll, D. Anielski, C. Bostedt, J. D. Bozek, L. Christensen, R. Coffee, S. De, P. Decleva, S. W. Epp, B. Erk et al., Femtosecond Photoelectron Diffraction on Laser-Aligned Molecules: Towards Time-Resolved Imaging of Molecular Structure, Phys. Rev. A 88, 061402(R) (2013).

[9] D. Rolles, R. Boll, M. Adolph, A. Aquila, C. Bostedt, J. D. Bozek, H. N. Chapman, R. Coffee, N. Coppola, P. Decleva et al., Femtosecond X-Ray Photoelectron Diffraction on Gas-Phase Dibromobenzene Molecules, J. Phys. B 47, 124035 (2014).

[10] R. Boll, A. Rouzée, M. Adolph, D. Anielski, A. Aquila, S. Bari, C. Bomme, C. Bostedt, J. D. Bozek, H. N. Chapman et al., Imaging Molecular Structure through Femtosecond Photoelectron Diffraction on Aligned and Oriented GasPhase Molecules, Faraday Discuss. 171, 57 (2014).

[11] F. Krasniqi, B. Najjari, L. Strüder, D. Rolles, A. Voitkiv, and J. Ullrich, Imaging Molecules from Within: Ultrafast Angström-Scale Structure Determination of Molecules via Photoelectron Holography Using Free-Electron Lasers, Phys. Rev. A 81, 033411 (2010).

[12] H. Fukuzawa, R. R. Lucchese, X.-J. Liu, K. Sakai, H. Iwayama, K. Nagaya, K. Kreidi, M. S. Schöffler, J. R. Harries, Y. Tamenori et al., Probing Molecular BondLength Using Molecular-Frame Photoelectron Angular Distributions, J. Chem. Phys. 150, 174306 (2019).

[13] M. Kazama, T. Fujikawa, N. Kishimoto, T. Mizuno, J. Adachi, and A. Yagishita, Photoelectron Diffraction from Single Oriented Molecules: Towards Ultrafast Structure Determination of Molecules Using X-Ray Free-Electron Laser, Phys. Rev. A 87, 063417 (2013).

[14] J. B. Williams, C. S. Trevisan, M. S. Schöffler, T. Jahnke, I. Bocharova, H. Kim, B. Ulrich, R. Wallauer, F. Sturm, T. N. Rescigno et al., Imaging Polyatomic Molecules in Three Dimensions Using Molecular Frame Photoelectron Angular Distributions, Phys. Rev. Lett. 108, 233002 (2012).

[15] H. Stapelfeldt and T. Seideman, Colloquium: Aligning Molecules with Strong Laser Pulses, Rev. Mod. Phys. 75, 543 (2003).

[16] M. Pitzer, M. Kunitski, A. S. Johnson, T. Jahnke, H. Sann, F. Sturm, L. Ph. H. Schmidt, H. Schmidt-Böcking, R. Dörner, J. Stohner, J. Kiedrowski, M. Reggelin, S. Marquardt, A. Schießer, R. Berger, and M. S. Schöffler, Direct Determination of Absolute Molecular Stereochemistry in Gas Phase by Coulomb Explosion Imaging, Science 341, 1096 (2013).

[17] T. Jahnke, Th. Weber, T. Osipov, A. L. Landers, O. Jagutzki, L. Ph. H. Schmidt, C. L. Cocke, M. H. Prior, H. SchmidtBöcking, and R. Dörner, Multicoincidence Studies of Photo and Auger Electrons from Fixed-In-Space Molecules Using the COLTRIMS Technique, J. Electron Spectrosc. Relat. Phenom. 141, 229 (2004).

[18] J. Ullrich, R. Moshammer, A. Dorn, R. Dörner, L. Ph. H. Schmidt, and H. Schmidt-Böcking, Recoil-Ion and Electron Momentum Spectroscopy: Reaction-Microscopes, Rep. Prog. Phys. 66, 1463 (2003).

[19] T. Tschentscher, C. Bressler, J. Grünert, A. Madsen, A. P. Mancuso, M. Meyer, A. Scherz, H. Sinn, and U. Zastrau, Photon Beam Transport and Scientific Instruments at the European XFEL, Appl. Sci. 7, 592 (2017).

[20] T. Weber, A. O. Czasch, O. Jagutzki, A. K. Müller, V. Mergel, A. Kheifets, E. Rotenberg, G. Meigs, M. H. Prior, S. Daveau et al., Complete Photo-Fragmentation of the Deuterium Molecule, Nature (London) 431, 437 (2004).

[21] Z. Bao, R. F. Fink, O. Travnikova, D. Céolin, S. Svensson, and M. N. Piancastelli, Detailed Theoretical and Experimental Description of Normal Auger Decay in $\mathrm{O}_{2}$, J. Phys. B 41, 125101 (2008).

[22] X.-J. Liu, C. Nicolas, M. Patanen, and C. Miron, Disentangling Auger Decays in $\mathrm{O}_{2}$ by Photoelectron-Ion Coincidences, Sci. Rep. 7, 2898 (2017).

[23] Ph. V. Demekhin, A. Ehresmann, and V. L. Sukhorukov, Single Center Method: A Computational Tool for Ionization 
and Electronic Excitation Studies of Molecules, J. Chem. Phys. 134, 024113 (2011).

[24] Ph. V. Demekhin, D. V. Omel'yanenko, B. M. Lagutin, V. L. Sukhorukov, L. Werner, A. Ehresmann, K.-H. Schartner, and H. Schmoranzer, Investigation of Photoionization and Photodissociation of an Oxygen Molecule by the Method of Coupled Differential Equations, Opt. Spectrosc. 102, 318 (2007).

[25] S. A. Galitskiy, A. N. Artemyev, K. Jänkälä, B. M. Lagutin, and $\mathrm{Ph}$. V. Demekhin, Hartree-Fock Calculation of the Differential Photoionization Cross Sections of Small Li Clusters, J. Chem. Phys. 142, 034306 (2015).
[26] See Supplemental Material at http://link.aps.org/ supplemental/10.1103/PhysRevX.10.021052 for molecular-frame photoelectron angular distributions of the second photoelectron (emitted during the $\mathrm{O}^{+} / \mathrm{O}^{+}$Coulomb explosion). The movie shows a sweep through different kinetic energy releases in the range of $28 \mathrm{eV}<\mathrm{KER}<36 \mathrm{eV}$. The red line is a fit using Legendre polynomials up to $l=9$. The internuclear distances $R$ have been obtained using the simple Coulomb explosion model (see Appendix B). The ion which is triply charged in the final state is located on the right. 